\title{
Evolving Fuzzy Neural Network Based on Uni-nullneuron to Identify Auction Fraud
}

\author{
${ }^{*}$ Paulo Vitor de Campos Souza ${ }^{a}$ and Edwin Lughofer ${ }^{a}$ and Augusto Junio Guimaraes ${ }^{b}$ \\ ${ }^{a}$ Dept. of Knowledge-Based Math. Systems, \\ Johannes Kepler University Linz, Altenberger Strasse, 69, 4040, Linz, Austria \\ paulo.de_campos_souza@jku.at, edwin.lughofer@jku.at \\ ${ }^{b}$ Information System Course, UNA University Center, Betim, Brazil, \\ augustojunioguimaraes@gmail.com
}

\begin{abstract}
The increase in transactions on the Internet related to the purchase of products or services can provide facilities for the parties involved in these acquisitions, but they also generate uncertainties and possibilities of attacks that can originate from fraud. This work seeks to explore and extract knowledge of auction fraud by using an evolving fuzzy neural network model based on n-uninorms. This new model uses a fuzzification technique based on Typicality and Eccentricity Data Analysis operators and a parallel processor for stream samples. To test the model in solving auction fraud problems, stateof-the-art neuro-fuzzy models were used to compare a public dataset on the topic. The results of the model proposed in this paper were superior to the other models evaluated (close to 96\% accuracy) in the test, and the fuzzy rules demonstrate the model's ability to extract knowledge.
\end{abstract}

Keywords: Parallel TEDA, Evolving Fuzzy Neural Network, Uni-Nullnorm, Auction Fraud

Intelligent models based on artificial intelligence have assumed a leading role in solving complex problems common in modern society. Intelligent methods can solve problems dynamically to optimize processes in industries, diagnostics of diseases, or actions related to transactions on the internet [21].

A current problem with the advent of the internet in people's lives is online shopping scams. Some sites promote interaction with users based on offers that are made about a product or service. This online auction is successful on a sales platform and encourages several users to buy products through bids that can be carried out remotely, according to standards established by the websites that offer this service. However, purchases involving high-value goods or rare items frequently suffer from fraud attempts. In general, online auction fraud has specific characteristics, such as faulty origin products, suspicious and repetitive bids on a given item, overvaluation of a product through constant bids, and users who register on the platform to cause problems [10]. These problems can make the experience of buying a product in the auction terrible, preventing new users from opting for this form of online purchase, in addition to discrediting the platform that offers these services [8]. Some researchers have built a database of existing factors in auctions to identify frauds during transactions to prevent problems like this from occurring [3]. These studies foster large areas of current research, and researchers from around the world work to identify the corresponding patterns and propose solutions to solve them, including approaches that use clustering and artificial intelligence [4].

Models based on artificial intelligence can act to assist in the detection of fraud in online auctions. In particular, a model that can combine the efficiency of training artificial neural networks and interpret fuzzy systems can solve this problem and extract knowledge of auction fraud through linguistic rules about the problem [7]. Fuzzy neural networks can process input data in fuzzy sets and, through training based on neural networks, provide answers to solve a problem with a high degree of interpretability since this model is composed of a fuzzy inference system, that through fuzzy operations, can represent the data in a linguistic and interpretable way [11].

This paper proposes a new evolving fuzzy neural network architecture based on a parallel fuzzification process that applies the concepts of Typicality and Eccentricity Data Analysis (TEDA) [17]. Logic neurons of the Uni-nullneuron (a logical neuron that now allows the use of a unineuron (using the uninorm operator) or a nullneuron (through the use of a nullnorm operator)) type proposed by de Campos Souza and Lughofer [12] 
are in the model's second layer, and training based on Extreme Learning Machine (ELM) [19]. The threelayer model has a Fuzzy Inference System connected to a Neural Aggregation Network represented in its third and last layer. This combination of factors allows the third layer of the model to provide the network's output with the answers about the frauds in the online auction and fuzzy rules that express the logical and interpretable relations present in the evaluated dataset. This paper's main highlight is linked to incorporating an evolving parallel approach in the network fuzzification method, besides one of the first models to extract knowledge of a problem related to auction fraud. Other intelligent models have already been used to solve these demands, but this paper presents the first neuro-fuzzy model to solve problems and extract the dataset's appropriate information.

The paper is organized as follows: In section 1, the literature will provide information about fuzzy neural networks and auction fraud. In section 2, the model used in this paper is presented, emphasizing its layers and training method. In section 3, the test characteristics to obtain auction fraud and the results obtained with their due arguments are presented. Finally, section 4 highlights the conclusions of the paper and possible future work.

\section{Literature Review}

\subsection{Fuzzy Neural Network}

The fuzzy neural networks (FNN) are constituted of fuzzy neurons, which are functional units that attach appropriate perspectives of processing with knowledge expertise and are beneficial for a parameter update in the intelligent models, besides facilitating the search of solutions to determine problems of distinct varieties. They can be recognized as multivariate non-linear alterations between hypercube units [24]. These networks are designed through a synergistic collaboration between fuzzy set theory and neural networks allowing a wide range of learning abilities, thus providing models that combine the uncertain information approach implemented by the fuzzy systems and the learning ability awarded the neural networks [24]. Therefore a Fuzzy neural network can be described as a fuzzy system trained by an algorithm implemented by an artificial neural network. Given this relationship, incorporating the neural network with the fuzzy logic appears to soften each of these techniques' insufficiency, addressing them as more cost-effective, robust, and straightforward to understand a problem. An evolving neural network updates its parameters as the model evaluates new samples. Its parameters better reflect the distinction of classes and facilitate the identification of pattern classification tasks.

These original models possess an architecture based on multi-layered networks, respectively, with specific purposes in the model. In de Campos Souza [15, 27] the FNN recognize pattern classification problem with the similar architecture of this paper. Other excellent cases are the pattern classification model that uses logical neurons proposed by de Campos Souza et al. to solve Optical Interconnection Network problems [14]. Most of the models listed above have differences in their architecture, where three and four-layer models stand out. Each of these layers' functions incorporates the concepts of fuzzy systems (fuzzification or defuzzification) and artificial neural networks. In the most significant models, the first layer is the one that partitions the input data, converting them into fuzzy logical neurons. Versions of fuzzy c-means, ANFIS, and clustering by the cloud are commonly implemented [11]. There are models responsible for pattern classification, fault prevention, and economic problems. Already in training algorithms, we stand out models based on backpropagation, genetic, evolutionary models, and extreme learning machine [11]. How the fuzzification occurs in the fuzzy neural network determines its architecture, and a reasonable approach, therefore, is proficient in performing a model with an optimized structure.

\subsection{Auction Fraud}

The constant digital revolution of the last decades has allowed the growth of E-commerce. Its main feature is to bridge the real and virtual world for the online shopping model. According to estimates by the United Nations Conference on Trade and Development - UNCTAD [23], E-commerce's global value in 2017 was around 29 trillion dollars. E-commerce is subdivided into several sales schemes [22], the most common of which are: Business to Business - (B2B), commercial transactions between companies; Business to Consumer - (B2C), which describes the activities of companies that serve end consumers with products and/or services; Consumer to Consumer - $(\mathrm{C} 2 \mathrm{C})$, in this format the relationship is from consumer to consumer, this is done through a platform that promotes the intermediation of the operation. In the three subdivisions mentioned, the practice of online auctions is present, in which an agent announces an item for sale, and other consumers or companies make bids to auction them off. The sites are only intermediaries to correspond to consumers, usually receiving a fixed fee or commission [22]. Online auctions have become very lucrative e-commerce. Despite all its benefits, there are also elevated costs because of the illegal acts [28]. 


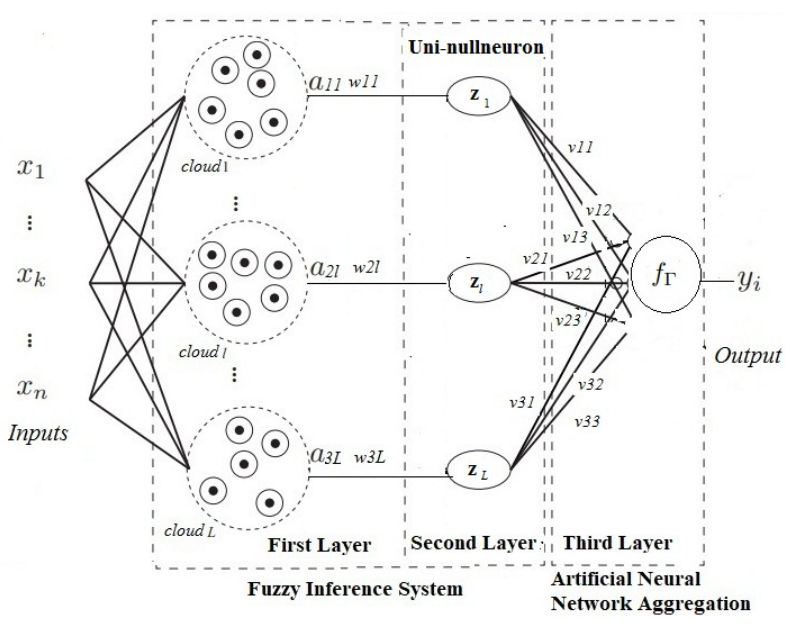

Figure 1: Architecture of the Fuzzy Neural Network proposed in this paper.

According to the FBI 2019 Internet Crime Report ${ }^{1}$, there were 467,361 complaints in 2019 - an average of almost 1,300 every day - and more than $\$ 3.5$ billion in losses to individual and commercial victims. The leading illicit practices in online auctions are, [28]: the announcement of goods coming from the black market or stolen; fraud that occurs during bidding time, such as Shill Bidding; and post-auction, such as the exaggerated collection of fees, insurance, and even the non-delivery of the acquired asset.

\section{Evolving Fuzzy Neural Network based on Parallel TEDA and Uni-nullNeuron}

Evolving fuzzy neural networks are models with an extraordinary degree of flexibility and independence that they acquire knowledge through the data offered for model training. This kind of approach helps the architecture's model, in particular, to define the logical structure that composes it. In this paper, the first layer of the model uses a Parallel algorithm based on input data density to develop logical neurons with Gaussian membership functions through the concept of uninullneurons. The model's architecture can be seen in Figure 1, and its other architectural aspects will be presented below.

\subsection{First Layer}

The first layer of the model proposed in this paper is responsible for the fuzzification process that allows Gaussian neurons' construction. This fuzzifica-

\footnotetext{
${ }^{1}$ FBI's Internet Crime Complaint Center (IC3) in its 2019 Internet Crime Report. Available in: https://pdf.ic3.gov/2019_IC3Report.pdf
}

tion process consists of applying a parallel technique based on the concepts of data density. The neuron construction occurs using a fuzzy set and weight defined in the interval between [0.1] based on an Online Feature weight technique. This facilitates the weight's determination according to the relevance of the feature to correctly identify the classes. More details about the technique can be seen in Lughofer [20]. The weight of this neuron can be defined by [12]:

$$
w_{i l}, i=1 \ldots N, l=1 \ldots L
$$

with $N$ the number of inputs (features) and $L$ represent the number of neurons. For each input variable $x_{i j}$, $L$ neurons are defined $A_{l j}, l=1, \ldots L$, whose activation functions are composed of membership functions in the corresponding neurons. A formal representation of the first layer neuron can be defined by:

$$
a_{j l}=\mu_{A_{l}}, j=1 \ldots n, l=1 \ldots L .
$$

where $a_{j l}$ represents the degree of association related to the inputs submitted to the model [12].

\subsection{Parallel Typicality and Eccentricity Data Analysis}

The fuzzification approach adopted in this paper was proposed by Gu et al. [17] and follow the concepts of exploring data characteristics to find clusters that can be adapted during the learning process as the model evaluates new data. This approach uses operators based on cumulative proximity of data, standard eccentricity, typicality so that clusters can be recursively calculated and, consequently, be adaptable to build clusters dynamically. In addition to these concepts, several data flow processors are involved, collaborating efficiently to achieve parallel computing to make the approach more efficient, robust and fast. Its central concept revolves around a fusion center capable of gathering the main information from processors that work in parts of the entire data flow and generate the clusters that represent the data. Angelov et al. defines the concepts of input variables as $\mathbf{x}=\left\{x_{1}, x_{2}, \ldots, x_{k}\right\} \in \mathbb{R}^{d}$, where the indices $k$ indicate the time instance at which the data point arrives. We can define the set of unique data point locations in time instance $k$ as $\mathbf{u}=\left\{u_{1}, u_{2}\right.$, $\left.\ldots, u_{Q},\right\} \in \mathbb{R}^{d}$ and the corresponding number of times that $f_{1}, f_{2}, \ldots, f_{Q}$ different data points occupy the same unique locations. Cumulative proximity based on these concepts can be written as [5]:

$$
\pi_{\kappa}\left(x_{i}\right)=\sum_{j=1}^{\kappa} d^{2}\left(x_{i}, x_{j}\right), i=1,2, \ldots, \kappa, \kappa>1
$$


where $\kappa$ is the number of the unique data samples and $d^{2}\left(u_{i}, u_{j}\right)$ express the distance measure between $x_{i}$ and $x_{j}$ (Euclidean). Other concepts used by the approach such as Standardized Eccentricity $\left(\varepsilon_{k}\right)$ and Typicality $\left(\tau_{k}\right)$ are defined respectively as [5]:

$$
\begin{gathered}
\varepsilon_{k}\left(x_{i}\right)=\frac{2 \pi_{\kappa}\left(x_{i}\right)}{\sum_{l=1}^{k} \pi_{\kappa}^{i}\left(x_{l}\right)}, i=1,2, \ldots, \kappa, \kappa>1 \\
\tau_{k}\left(x_{i}\right)=\frac{\frac{1}{\pi_{\kappa}\left(x_{l}\right)}}{\sum_{l=1}^{k} \frac{1}{\pi_{\kappa}\left(x_{l}\right)}}, i=1,2, \ldots, \kappa, \kappa>1
\end{gathered}
$$

These metrics can be updated recursively as the model evaluates new samples. The recursive form of these TEDA operators can be seen in $\mathrm{Gu}$ et al. [17]. The parallel TEDA approach has some steps for constructing clusters, where the data samples arrive continuously and are divided into blocks and sent to different data flow processors [17]. First, the data blocks are sent to the data flow processor, being processed separately, where the results of the current processing will be influenced only by the previous data blocks processed within the same processor. The approach has two stages where the first is to update clusters and parameters within the individual data stream separately, and the second step is to merge the separate clustering results from all existing data streams, called cluster merging [17].

All data blocks from the data stream are processed separately on their corresponding processors in the separate processing step. At this stage, the standardized eccentricities of all samples are evaluated. Also, the conditions for associating a sample with a given cluster are checked based on this expression [17]:

$$
\operatorname{IF}\left(\varepsilon_{k+1}^{i, c}\left(x_{k+1}^{i}\right) \leq \varepsilon_{o}\right) \quad \operatorname{THEN}\left(x_{k+1}^{i} \in c\right)
$$

where $c$ represent the cluster, $\varepsilon_{o}$ is the intra-cluster variance (defined as 5 like in Gu et al. [17]) and $x_{k+1}^{i}$ is the new data sample. When this condition is met, the sample is assigned to this cluster, and the parameters of the cluster are updated recursively. If the conditions are not met, a new cluster is formed, and its parameters are duly calculated [17].

The approach is also capable of removing obsolete clusters that can harm the grouping of data. After this cleaning of unnecessary clusters, the data flow processor will be ready for the next data sample and start a new round of data processing and parameter updating. Finally, in this stage, mergers of clusters that can be grouped are carried out, and the final result results from the fuzzification process. The following situation assesses the merge condition of a cluster [17]:

$$
\begin{array}{r}
\operatorname{IF}\left(\varepsilon_{l}\left(\mu_{j}\right) \leq \varepsilon_{o}\right) \text { AND }\left(\varepsilon_{l}\left(\mu_{l}\right) \leq \varepsilon_{o}\right) \\
\text { THEN (The } \left.j^{\text {th }} \text { and } l^{\text {th }} \text { clusters should be merged. }\right)
\end{array}
$$

where $\mu_{j}$ and $\mu_{l}$ are the centers of the $j^{t h}$ and $l^{\text {th }}$ clusters. Therefore, this fuzzification approach and, consequently, the model have two parameters: the block value and the number of processors.

\subsection{Second Layer}

The second layer comprises fuzzy logic neurons that use an operator called uni-nullnorm [1]. This type of operator acts similarly to uninorms [29] and nullnorms [9] capable of using traditional fuzzy operators (t-norms and t-conorms) more flexible. An example of Uninorm is presented as follow:

$$
U(x, y, g)=\left\{\begin{array}{c}
g T\left(\frac{x}{o}, \frac{y}{g}\right), \text { if } y \in[0, g] \\
g+(1-g) S\left(\frac{x-g}{1-g}, \frac{y-g}{1-g}\right), \text { if } y \in(g, 1]
\end{array}\right.
$$

A particular example of n-uninorm called 2-uninorms is used in this article and can be represented as a function $N^{u n}:[0,1]^{2} \rightarrow[0,1]$, being a commutative binary function with $g, u, \beta \in[0,1]$ with $0 \leq g \leq \beta \leq u \leq 1$ and $0<\beta<1$. Therefore, $\{g, u\}_{\beta}$ is the definition of a 2-neutral element of $N^{u n}$ if it satisfies [1]:

$$
\begin{aligned}
& -N^{u n}(g, \mathrm{x})=\mathrm{x} \text { for all } \mathrm{x} \leq \beta \\
& \text { - } N^{u n}(u, \mathrm{x})=\mathrm{x} \text { for all } \mathrm{x} \geq \beta
\end{aligned}
$$

The 2-uninorm, $\left(N^{u n}=[0,1]^{2} \rightarrow[0,1]\right)$ is commutative, associative and has $\{g, u\}_{\beta}[1]$. The $N^{u n}$ operator can act as a uninorm if $g=\beta=u$, just as it can turn into a nullnorm if $g=0$ and $u=0$. This behavior of the 2-uninorm operator is flexible relative to its operators, and in this paper, if $0<g<\beta<u<1$, it can be written as follows [30]:

$$
N^{u n}(x, y, \beta, g, u)=\left\{\begin{array}{c}
\beta U_{1}\left(\frac{x}{\beta}, \frac{y}{\beta}\right), \text { if } x, y \in[0, \beta] \\
\beta+(1-\beta) U_{2}\left(\frac{x-\beta}{1-\beta}, \frac{y-\beta}{1-\beta}\right) \\
, \text { if } x, y \in(\beta, 1]
\end{array}\right.
$$

where $U_{1}$ is a uninorm (Eq. 8) with a neural element (identity) $=\frac{g}{\beta}$ and $U_{2}$ is a uninorm (Eq. 8) using $\left(\frac{u-\beta}{1-\beta}\right)$ like as neutral element. This property of Uninullnorms (using 2-uninorm) allows this operator to 
behave as uninorms or nullnorms, allowing the formed fuzzy rules to be flexible concerning the logical connectors of the rules (AND / OR).

The Uni-nullneuron proposed by de Campos Souza and Lughofer [12] operate fuzzy sets through operations that aggregate them, transforming them into unique values. Thus, the Uni-nullneuron used in this paper is represented by:

$$
z=U N I^{N U L}(w, a, \beta, g, u)=N^{u n_{i=1}^{n}} p\left(w_{i}, a_{i}, \beta, g, u\right)
$$

where $p$, is a function called conditional transformation. The $p$ function can provide the aggregation of the neuron input values in two levels, where the first level calculates the weighting of the input with its respective weight and the second level performs a global aggregation. This function is represented by [12]:

$$
p(w, a, \beta, g, u)=\left\{\begin{array}{c}
w a+\bar{w} \frac{g}{\beta}, \text { if } U_{1} \\
w a+\bar{w} \frac{u-\beta}{1-\beta}, \text { if } U_{2}
\end{array}\right.
$$

where $\bar{w}$ represents the complement of $w$.

\subsection{Third Layer}

The third layer of the model represents a neural aggregation network with a neuron (also called Singleton) responsible for the model's final responses. The neuron used a linear activation function, and to process the model's binary outputs, it uses a signal function, which transforms the output value into a value of -1 or 1 , depending on the final operation value of this model. This representation can be defined by [12]:

$$
y=\Omega\left(\sum_{j=0}^{l} f_{\Gamma}\left(z_{j}, v_{j}\right)\right)
$$

where $z_{0}=1, v_{0}$ is the bias, and $z_{j}$ and $v_{j}, j=1, \ldots, l$ are the output of each fuzzy neuron of the second layer and their corresponding weight, respectively. $f_{\Gamma}$ represents the linear activation function and $\Omega$ the signal function [12].

\subsection{Training and Model Interpretability}

The artificial neural networks training is carried out through the concept of Extreme Learning Machine [19], and the definition of weights of the neural network is expressed by [12]:

$$
\vec{v}_{k}=Z^{+} \vec{y}_{m} \quad \forall m=1, \ldots, 2
$$

where $m$ is the number of classes, $Z^{+}=Z^{T} Z$ is the pseudo-inverse of the Moore-Penrose matrix [2] of $Z$ (output of the second layer).

For new incoming on-line stream samples was used the recursive weighted least squares (RWLS) approach [18], with formulas for updating the $\vec{v}_{k}$ and can be expressed as follow:

$$
\begin{gathered}
\eta=\vec{z}^{t} Q^{t-1}\left(\psi+\left(\vec{z}^{t}\right)^{T} Q^{t-1} \vec{z}^{t}\right)^{-1} \\
Q^{t}=\left(\mathrm{I}_{L^{t}}-\eta^{T} \vec{z}^{t}\right) \psi^{-1} Q^{t-1} \\
\vec{v}_{k}^{t}=\vec{v}_{k}^{t-1}+\eta^{T}\left(y_{k}^{t}-\vec{z}^{t} \vec{v}_{k}^{t-1}\right)
\end{gathered}
$$

where the index $k$ again denotes the class index $k=$ $1, \ldots, C . \vec{z}^{t}$ denotes the regressor vector of the current sample, $\eta$ is the current kalman gain vector, $I_{L_{s}^{t}}$ is an identity matrix based on the number of neurons in the second layer, $\left.\left.L_{s}^{t} \times L_{s}^{t} ; \psi \in\right] 0,1\right]$ denotes forgetting factor (1 per default). $Q$ denotes the inverse Hessian matrix $Q=\left(Z_{\text {sel }}^{T} Z_{\text {sel }}\right)^{-1}$ and is set initially as $\omega I_{L_{s}^{t}}$, where $\omega=1000$ [25]. This matrix is directly and incrementally updated by the second equation above without requiring (time-consuming and possibly unpredictable) matrices re-inversion. The model's interpretability criteria are based on two factors: the fuzzy rules generated from the model. Uni-nullneuron can generate rules in the following format [12]:

$$
\begin{array}{r}
\text { Rule }_{1}: \text { If } x_{i 1} \text { is } A_{1}^{1} \text { with impact } w_{11} \ldots \\
\text { and } / \operatorname{or}_{(g, u, \beta)} x_{i N} \text { is } A_{1}^{N} \text { with impact } w_{N 1} \ldots \\
\text { Then } y_{1} \text { is } v_{1} \\
\text { Rule }_{2}: \text { If } x_{i 1} \text { is } A_{M}^{1} \text { with impact } w_{1 L} \ldots \\
\text { and } / \text { or }_{(g, u, \beta)} x_{i 2} \text { is } A_{M}^{N} \text { with impact } w_{N L} \ldots \\
\text { Then } y_{L} \text { is } v_{L}
\end{array}
$$

\section{Auction fraud testing}

The tests that were performed, models, and the dataset will be presented below.

\subsection{Dataset features}

This work aims to analyze the Shill Bidding bid fraud. The analyzed dataset was collected by [3] and made available in the Machine Learning Repository - UCI 2 . The data corresponds to fraudulent bids on one of the largest online auction sites, eBay. The collected database [3], presents completed iPhone 7 auctions for three months (March to June 2017). The original dataset has 12 input features, but for the studies carried out in this paper, the dimensions related to personal

\footnotetext{
${ }^{2}$ archive.ics.uci.edu/ml/datasets/Shill+Bidding+Dataset
} 
identifications (Record ID, Auction ID, and Bidder ID because they are identification values and not values collected in the experiments) were removed. The remaining nine dimensions are Bidder Tendency, Bidding Ratio, Successive Outbidding, Last Bidding, Auction Bids, Auction Starting Price, Early Bidding, Winning Ratio, Auction Duration, and Class (1 to normal and -1 to Fraud).

\subsection{Models and hyperparameters}

EFDDC- The evolving model uses data density-based clustering based on EDA operators and nullneuron. The model's training is based on the ELM and bolasso technique to select the best neurons. The model parameters are $\rho=0.01, b t=16$ and $\lambda=0.7$ [16].

EFNHN- The model combines an evolving fuzzification technique based on data density (Autonomous Data Partitioning), training based on ELM, and Unineurons. The Defuzzification process is based on an Artificial Hydrocarbon network. The model parameter is the Learning rate $=0.1$ [26].

EFNN - The evolving neural network model uses the Self-Organised direction aware for fuzzification, unineurons, ELM, and pruning technique. The only parameter used in the model is the grid size $=3$ [13]

\subsection{Results}

The results of the evaluation of the trend lines are presented in Figure 2. This evaluation was carried out with $10 \%$ of the total samples (632) for training and the rest $(5,689)$ to evaluate the model. For the experiments, the block values $=40$ and the number of processors $=$ 5 were considered ${ }^{3}$. These lines were calculated using the following criteria: [6]:

$$
A C C(K+1)=\frac{A C C(K) * K+I_{\hat{y}=y}}{K+1},
$$

where ACC:

$$
A C C=\frac{T P+T N}{T P+F N+T N+F P} * 100 .
$$

where $T P=$ true positive, $T N=$ true negative, $F N=$ false negative and $F P=$ false positive.

The results presented in Figure 2 prove that the model proposed in this paper can correctly identify the evaluated samples. The comparison made with the state-ofthe-art models allows us to verify that the model can identify fraud behaviors in the auctions. This approach

\footnotetext{
${ }^{3}$ The values were defined through a cross-validation procedure for block values $=[50,60,70,80,90]$ and the number of processors $=[3,4,5,6,7]$.
}

promises to work with large data streams with a high degree of identification about whether or not there is a fraud occurring. This behavior can prevent high financial amounts from being protected in online shopping transactions. In this experiment, ten fuzzy rules were generated to solve the auction fraud problem. These ten rules ${ }^{4}$ allow the extraction of knowledge disseminated and facilitate intelligent systems to assist this process during online transactions. The following are examples of rules that facilitate the identification of Fraud or normal behavior in auctions.

Rule 1. If (Bidder Tendency is Very High) with impact 0.02 and (Bidding Ratio is Very Small) with impact 0.08 and (Successive Outbidding is Medium) with impact 1.00 and (Last Bidding is Medium) with impact 0.00 and (Auction Bids is Very Small) with impact 0.00 and (Auction Starting Price is Very Small) with impact 0.00 and (Early Bidding is Extremely High) with impact 0.00 and (Winning Ratio is Very Small) with impact 0.02 and (Auction Duration is Very Small) with impact 0.00 and then (bid is Normal).

Rule 2. If (Bidder Tendency is Extremely High) with impact 0.02 or (Bidding Ratio is Very Small) with impact 0.08 or (Successive Outbidding is Small) with impact 1.00 or (Last Bidding is Small) with impact 0.00 or (Auction Bids is Very Small) with impact 0.00 or (Auction Starting Price is Very Small) with impact 0.00 or (Early Bidding is Very High) with impact 0.00 or (Winning Ratio is Very Small) with impact 0.02 or (Auction Duration is Very Small) with impact 0.00 and then (bid is Fraud).

The use of weights correlated to the relevance of the features allows these extracted rules to be simplified. Zero-weight antecedents can be discarded, allowing fuzzy rules to be rewritten. They are presented below:

Rule 1. If (Bidder Tendency is Very High) with impact 0.02 and (Bidding Ratio is Very Small) with impact 0.08 and (Successive Outbidding is Medium) with impact 1.00 and (Winning Ratio is Very Small) with impact 0.02 then (bid is Normal).

Rule 2. If (Bidder Tendency is Extremely High) with impact 0.02 or (Bidding Ratio is Very Small) with impact 0.08 or (Successive Outbidding is Small) with impact 1.00 or (Winning Ratio is Very Small) with impact 0.02 then (bid is Fraud).

After the rules are simplified, it is possible to recognize the most relevant factors for identifying classes.

\footnotetext{
${ }^{4}$ The linguistic terms are generated by the Gaussian functions in the first layer.
} 


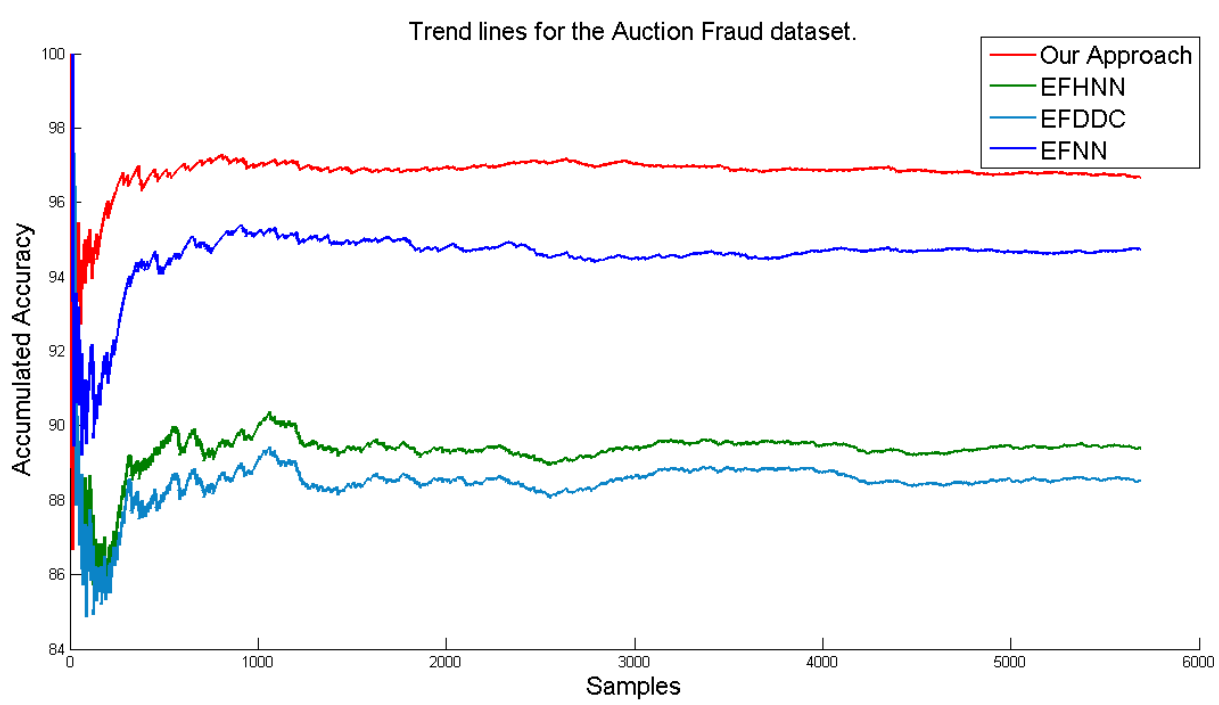

Figure 2: Trend lines for the Auction Fraud dataset.

Noteworthy are the features of Successive Outbidding accompanied by Bidding Ratio. The other fuzzy rules also have other correlations, but in all cases, the dimensions highlighted can identify with a high degree of assertiveness the possible actions in an auction.

\section{Conclusion}

The model proposed in this paper achieved the best results compared to the state-of-the-art model in identifying auction fraud. That is due to an efficient evolving fuzzification technique that was able to identify the characteristics present in the dataset correctly. Simultaneously, it acted in the evolution of the model parameters simply and efficiently. The extraction of fuzzy rules (whereby their dimensionality has been reduced) identify which dimensions of the problem are most significant for identifying frauds. This type of approach corroborates the high ability to understand a problem and its possible use to incorporate new techniques in identifying auction fraud that can generate financial inconvenience for those who use this technique.

Possible future work can be done to create new training techniques for evolving the model and using other neural structures to improve the accuracy of the model. Other datasets with different configurations and evaluations on auction fraud are also encouraged to verify the model's adaptability in solving this nature's problems.

\section{Acknowledgement}

The authors acknowledge the support by the Austrian Science Fund (FWF): contract number P32272-N38, acronym IL-EFS.

\section{References}

[1] P. Akella, Structure of n-uninorms, Fuzzy Sets and Systems 158 (15) (2007) 1631-1651.

[2] A. Albert, Regression and the Moore-Penrose pseudoinverse, Elsevier, 1972.

[3] A. Alzahrani, S. Sadaoui, Scraping and preprocessing commercial auction data for fraud classification, arXiv preprint arXiv:1806.00656.

[4] A. Alzahrani, S. Sadaoui, Clustering and labeling auction fraud data, in: Data Management, Analytics and Innovation, Springer, 2020, pp. 269283.

[5] P. Angelov, X. Gu, D. Kangin, Empirical data analytics, International Journal of Intelligent Systems 32 (12) (2017) 1261-1284.

[6] A. Bifet, G. Holmes, R. Kirkby, B. Pfahringer, MOA: Massive online analysis, Journal of Machine Learning Research 11 (2010) 1601-1604.

[7] J. Buckley, Y. Hayashi, Fuzzy neural networks: A survey, Fuzzy sets and systems 66 (1) (1994) $1-13$.

[8] M. Button, C. M. Nicholls, J. Kerr, R. Owen, Online frauds: Learning from victims why they fall for these scams, Australian \& New Zealand journal of criminology 47 (3) (2014) 391-408. 
[9] T. Calvo, B. De Baets, J. Fodor, The functional equations of frank and alsina for uninorms and nullnorms, Fuzzy Sets and Systems 120 (3) (2001) 385-394.

[10] C. E. H. Chua, J. Wareham, Fighting internet auction fraud: An assessment and proposal, Computer 37 (10) (2004) 31-37.

[11] P. V. de Campos Souza, Fuzzy neural networks and neuro-fuzzy networks: A review the main techniques and applications used in the literature, Applied Soft Computing 92 (2020) 106275.

[12] P. V. de Campos Souza, E. Lughofer, An advanced interpretable fuzzy neural network model based on uni-nullneuron constructed from nuninorms, Fuzzy Sets and Systems.

[13] P. V. de Campos Souza, T. S. Rezende, A. J. Guimaraes, V. S. Araujo, L. O. Batista, G. A. da Silva, V. J. Silva Araujo, Evolving fuzzy neural networks to aid in the construction of systems specialists in cyber attacks, Journal of Intelligent \& Fuzzy Systems 36 (6) (2019) 6743-6763.

[14] P. V. de Campos Souza, E. A. Soares, A. J. Guimarães, V. S. Araujo, V. J. S. Araujo, T. S. Rezende, Autonomous data density pruning fuzzy neural network for optical interconnection network, Evolving Systems (2020) 1-13.

[15] P. V. de Campos Souza, L. C. B. Torres, A. J. Guimaraes, V. S. Araujo, V. J. S. Araujo, T. S. Rezende, Data density-based clustering for regularized fuzzy neural networks based on nullneurons and robust activation function, Soft Computing 23 (2019) 12475-12489.

[16] P. V. de Campos Souza, L. C. B. Torres, A. J. Guimaraes, V. S. Araujo, V. J. S. Araujo, T. S. Rezende, Data density-based clustering for regularized fuzzy neural networks based on nullneurons and robust activation function, Soft Computing 23 (23) (2019) 12475-12489.

[17] X. Gu, P. P. Angelov, G. Gutierrez, J. A. Iglesias, A. Sanchis, Parallel computing teda for high frequency streaming data clustering, in: P. Angelov, Y. Manolopoulos, L. Iliadis, A. Roy, M. Vellasco (Eds.), Advances in Big Data, Springer International Publishing, Cham, 2017, pp. 238-253.

[18] G.-B. Huang, L. Chen, C. K. Siew, et al., Universal approximation using incremental constructive feedforward networks with random hidden nodes, IEEE Trans. Neural Networks 17 (4) (2006) 879-892.
[19] G.-B. Huang, Q.-Y. Zhu, C.-K. Siew, Extreme learning machine: theory and applications, Neurocomputing 70 (1-3) (2006) 489-501.

[20] E. Lughofer, On-line incremental feature weighting in evolving fuzzy classifiers, Fuzzy Sets and Systems 163 (1) (2011) 1-23.

[21] R. Mitchell, J. Michalski, T. Carbonell, An artificial intelligence approach, Springer, 2013.

[22] R. Nemat, Taking a look at different types of ecommerce, World Applied Programming 1 (2) (2011) 100-104

[23] U. N. C. on Trade, D. (UNCTAD), Digital economy report 2019: Value creation and capture: implications for developing countries (2019).

[24] W. Pedrycz, Neurocomputations in relational systems, IEEE Transactions on Pattern Analysis \& Machine Intelligence 13 (3) (1991) 289-297.

[25] R. Rosa, F. Gomide, D. Dovzan, I. Skrjanc, Evolving neural network with extreme learning for system modeling, in: 2014 IEEE Conference on Evolving and Adaptive Intelligent Systems (EAIS), 2014, pp. 1-7.

[26] P. Souza, H. Ponce, E. Lughofer, Evolving fuzzy neural hydrocarbon networks: A model based on organic compounds, Knowledge-Based Systems 203 (2020) 106099.

[27] P. V. C. Souza, Regularized fuzzy neural networks for pattern classification problems, International Journal of Applied Engineering Research 13 (5) (2018) 2985-2991.

[28] J. Trevathan, Getting into the mind of an "inauction" fraud perpetrator, Computer Science Review 27 (2018) 1-15.

[29] R. R. Yager, A. Rybalov, Uninorm aggregation operators, Fuzzy sets and systems 80 (1) (1996) 111-120.

[30] H. Zhou, X. Liu, Characterizations of (u2,n)implications generated by 2-uninorms and fuzzy negations from the point of view of material implication, Fuzzy Sets and Systems 378 (2020) 79-102. 